Check for updates

1 Queen Mary University of London, UK

@MaryVenn4

m.l.venn@qmul.ac.uk

Cite this as: BMJ 2020;370:m3466 http://dx.doi.org/10.1136/bmj.m3466

Published: 17 September 2020
PARTNERSHIP IN PRACTICE

\section{Surgery during covid-19: co-producing patient resources}

Surgical patients are facing new territory. On the one hand they hear that elective surgery carries an increased risk of getting covid-19, on the other, that delaying surgery may cause problems, and that operations can be done safely when precautions are taken. In this article, Mary Venn, surgical research fellow, and Carrie Tierney Weir, a patient with inflammatory bowel disease, describe how their collaborative group, CovidSurg, has worked with patients to rapidly produce information resources about the risks of surgery during the pandemic.

Mary L Venn, ${ }^{1}$ Carrie Tierney Weir

\section{Mary Venn: Covid-19 research changing the pace in patient engagement}

CovidSurg is an international collaborative of surgeons and anaesthetists capturing and sharing data to improve clinical care. The first data from our CovidSurg cohort study, from 235 hospitals in 24 countries, identified a $24 \%$ overall mortality rate in patients with perioperative SARS-CoV-2 infections. ${ }^{1}$ Postoperative pulmonary complications in these patients were far more frequent than before the pandemic, occurring in $51 \%$.

These findings highlighted the need to discuss covid-related complications with patients as part of the surgical consent process. They also prompted us to immediately draw up draft information for patients due to undergo surgery. We quickly established a patient advisory group to appraise and develop information resources, and a web based platform for patients and carers. We included patients with benign or malignant conditions and a few who had experience of hospital care during the pandemic.

Our opening submission to the group was a shiny eight page patient information booklet that felt "almost finished." We expected a stamp of approval on our smart graphics and catchy titles. Constructive critique included: "Don't use 'era'," "what do you mean by recovery period?," and "the calendar icon has an 8 on it, does that mean eight days?" It took some humility to watch the booklet taken apart and rebuilt to include additional information, with more clarity.

\section{Carrie Tierney Weir: knowledge and experience to share}

When my surgeon told me I needed major bowel surgery for complications of inflammatory bowel disease, I had difficulty concentrating and barely retained any of the information provided during the consultation. Verbal discussion at a consultation should be supported by detailed information that patients can read and digest in the familiarity of their home.
Our patient advisory group included a mix of patients and carers, led by a surgical representative from CovidSurg. We met by video conference and exchanged views about the information we needed to make a decision about undergoing surgery. It soon became clear we had common views as well as substantial knowledge and experience to share. The booklet is intended to be used in conjunction with other communication methods, and not to replace conversations with surgeons.

Together the team identified key questions:

- Why are operations being cancelled?

- How are patients being prioritised for surgery?

- What are hospitals doing to keep patients safe?

- What can patients do to stay safe and best prepare for an operation?

- What will happen if I need emergency surgical care?

\section{Collaborating to produce new resources}

Patients wanted the risks of perioperative covid-19 quantified clearly in numbers. They also wanted detailed information about the measures hospitals are taking to keep them safe. Their questions extended beyond the information that we (surgeons) had anticipated, and included questions about whether visitors would be allowed, how patients might communicate with friends and family, and whether they might be discharged home sooner than usual.

Working together led to a higher quality and truly co-produced new patient information booklet to answer patient questions and provide the latest evidence about patient outcomes with perioperative SARS-CoV-2 infection. Our booklet is designed for any patient undergoing any type of surgery during the pandemic. We produced versions for people with learning disabilities and for those with dyslexia, and our research dissemination team translated the booklet into 16 languages. 
In addition we have co-produced lay summaries of all CovidSurg research, which are available on our website bit.ly/researchexplained. The patient information booklet is available at bit.ly/surgeryduringcovid.

\section{Advantages and challenges of virtual exchange}

During the covid-19 pandemic, we clinicians have learned to host webinars and publish research material online. Members of the patient advisory group valued being able to communicate digitally and work safely from their own homes. They described feeling valued and uninhibited by the less formal setting, and more able to contribute using screen shares.

As digital working increases, it will be necessary to assist potential group members to set up teleconferencing software, understand online data sharing, consent to participate, and offer the option to do so without being "on camera."

\section{Next steps}

Our next project; GlobalSurg-CovidSurg Week, is an international prospective cohort study to determine the optimal timing of surgery following SARS-CoV-2 infection and assess key global surgical outcomes such as mortality. We engaged the patient advisory group at the study design stage, so this study will answer questions important to patients as well as healthcare providers. To date, 1850 hospitals in 132 countries have expressed an interest in participating and registration is still open (globalsurg.org/surgweek/).

For more information, please visit bit.ly/surgerycovid

Competing interests: None declared.

Acknowledgments: Lesley Booth, Daoud Chaudhry, Karolin Kroese, Maria Picciochi, Abhinav Tiwari, April Roslani, Raza Sayyed, Gaetano Gallo, Charles Knowles, Haytham Kaafarani, Elizabeth Li, Joana Simões, James Glasbey, Dmitri Nepogodiev, Aneel Bhangu.

Provenance and peer review: not commissioned; not externally peer reviewed.

Patient consent obtained

See the full version of this article at https://blogs.bmj.com/bmj/2020/08/28/rapid-co-creation-of-information-resources-for-surgical-patients-during-covid-19/

If you would like to contribute to the Partnership in Practice series, please contact Tessa Richards trichards@bmj.com 